\title{
LEPTOSPIROSE HUMANA: UMA REVISÃO SOBRE A DOENÇA E OS FATORES DE RISCO ASSOCIADOS À ZONA RURAL
}

Recebido: 19/06/2017

Aceito: 09/11/2017

\author{
MACHADO, Gilmar Batista "; \\ SEIXAS NETO, Amilton Clair ${ }^{2}$; \\ DEWES, Caroline ${ }^{3}$; \\ FORTES, Tanise Pacheco ${ }^{4}$; \\ PACHECO, Paula Soares ${ }^{5}$; \\ FREITAS, Laís Santos ${ }^{6}$; \\ FELIX, Samuel Rodrigues ${ }^{2}$; \\ SILVA, Éverton Fagonde da ${ }^{7}$.
}

\begin{abstract}
${ }^{1}$ Doutorando do Programa de Pós-Graduação em Veterinária/UFPEL; ${ }^{2}$ PNPD do Programa de Pós-Graduação em Veterinária/UFPEL; ${ }^{3}$ Doutoranda do Programa de Pós-Graduação em Veterinária/UFPEL; ${ }^{4}$ Doutora em Veterinária; ${ }^{5}$ Graduanda em Medicina Veterinária/UFPEL; ${ }^{6}$ Mestranda do Programa de Pós-Graduação em Veterinária; ${ }^{7}$ Professor Doutor, Faculdade de Veterinária, UFPEL.
\end{abstract}

\section{RESUMO}

A leptospirose é uma zoonose de distribuição mundial cuja transmissão às espécies suscetíveis ocorre pelo contato direto ou indireto com indivíduos infectados. A moléstia é causada por bactérias do gênero Leptospira, acomete tanto humanos quanto animais. No meio rural, o risco dos humanos em adquirir a doença é maior do que para os moradores do meio urbano, principalmente pelo contato com animais domésticos, sinantrópicos e silvestres. Nesse contexto, este trabalho objetivou revisar os principais estudos epidemiológicos sobre a leptospirose na área rural do estado do Rio Grande do Sul (RS) nos últimos vinte e cinco anos, inferindo sobre a influência das atividades agropecuárias nos casos da doença em humanos. Evidencia-se que as populações rurais no RS, principalmente as localizadas na região sul e central estão mais expostas e possuem maiores chances de contrair a leptospirose, quando comparadas com a zona urbana. No RS, as atividades que envolvem longos períodos de trabalho em contato com a água e a indisponibilidade, na maioria das vezes, de equipamentos de proteção individual (EPI), aumentam o risco para a contaminação com o agente. Entre os animais domésticos, destacam-se os caninos e bovinos como importantes transmissores da enfermidade para os humanos no meio rural. Além disso, falhas no saneamento e coleta de lixo favorecem a presença de roedores nas propriedades, elevando o risco para a ocorrência da doença. Assim, medidas como a reeducação, a informação sobre os principais fatores de riscos da doença e o uso de EPI podem ser utilizadas para prevenir casos graves de leptospirose no meio rural do RS.

Palavras-chave: Leptospira. Saúde Pública. Doença Ocupacional. Zoonose. 


\section{INTRODUÇÃO}

A leptospirose é uma doença bacteriana, zoonótica de distribuição mundial, endêmica em países tropicais e subtropicais (DREYFUS et al., 2016). A transmissão ocorre através da exposição, direta ou indireta à urina ou tecidos abortados de animais infectados, pelo contato direto com animais portadores, contato com água ou solo contaminados (HARTSKEERL et al., 2011). A doença é causada por bactérias do gênero Leptospira que contém 22 espécies e mais de 300 sorotipos descritos (DREYFUS et al., 2016).

Nas cidades brasileiras, sua ocorrência está relacionada a condições inadequadas de habitação, permitindo o contato dos humanos com águas ou solos contaminados, principalmente em períodos de chuvas e enchentes, favorecendo a infecção (FONTES et al., 2010). A leptospirose foi descrita pela primeira vez em 1880, no Cairo, por Larrey, no entanto, foi em 1886 que Weil descreveu minuciosamente quatro casos clínicos em humanos (SIMÕES et al., 2016). No Brasil, os primeiros relatos sobre leptospirose foram publicados no Rio de Janeiro, em 1917, por Aragão, sobre "A presença do Spirochaeta icterohaemorrhagiae nos ratos do Rio de Janeiro" (OLIVEIRA, 2008).

A leptospirose possui um amplo espectro de manifestações clínicas, que vão desde a infecção subclínica, podendo apresentar doença febril anictérica, com ou sem meningite, e uveíte; até as formas graves com elevada letalidade, com icterícia, insuficiência renal e hemorragia (doença de Weil) (KO et al., 2009) e síndrome hemorrágica pulmonar (GULATI; GULATI, 2012). De acordo com Costa et al. (2015), a estimativa é que ocorra mais de 1 milhão de casos, que ocasionam mais de 58 mil mortes anualmente em todo o mundo. No estado do Rio Grande do Sul, mais de 400 casos são notificados anualmente, e estima-se que a real incidência da enfermidade seja mais elevada, especialmente em populações vulneráveis, onde a leptospirose é comumente diagnosticada e não declarada (SCHNEIDER et al., 2015).

Neste contexto, o presente trabalho teve como objetivo revisar a bibliografia disponível sobre leptospirose na zona rural do Rio Grande do Sul nos últimos 25 anos, ressaltando a associação dos animais domésticos com a doença em humanos. 


\section{A BACTÉRIA}

A leptospirose é causada por bactérias do gênero Leptospira, que acomete humanos e animais, os quais participam da cadeia epidemiológica como doentes, portadores assintomáticos e reservatórios (FIGUEIREDO et al., 2001). A organização estrutural e a composição química das leptospiras são semelhantes às de bactérias Gram-negativas, como a membrana externa que envolve toda a célula, os filamentos axiais denominados de flagelos periplasmáticos e os cilindros protoplasmáticos, que incluem a membrana celular e a capa de peptidioglicano da parede celular (SIMÕES et al., 2016).

As leptospiras são micro-organismos helicoidais que medem de 6 a $25 \mu \mathrm{m}$ de comprimento por 0,2 $\mu \mathrm{m}$ de diâmetro (BHARTI et al., 2003). São aeróbios estritos que apresentam uma ou ambas as extremidades curvadas ou em forma de gancho, dotados de grande motilidade conferida por um axóstilo (SIMÕES et al., 2016). Apresentam crescimento ótimo em uma faixa de temperatura entre 28 a $30{ }^{\circ} \mathrm{C}$, possuem multiplicação lenta e são exigentes no que se refere a meios de cultivo, sendo rotineiramente suplementados com soro de coelho ou suplementos comerciais (CAMERON, 2015).

\section{IMUNIDADE}

As leptospiras penetram no hospedeiro através das mucosas e da pele, íntegras ou lesadas. Após vencer as barreiras de porta de entrada, multiplica-se no espaço intersticial e nos humores orgânicos (sangue, linfa e líquor), caracterizando um quadro agudo septicêmico denominado de leptospiremia (SIMÕES et al., 2016).

A ausência de fagócitos no filtrado glomerular permite a multiplicação destes microrganismos nos túbulos contorcidos renais formando microcolônias. Desta localização, as leptospiras passam a ser eliminadas através da urina (leptospirúria) por períodos variáveis, de dias a anos. Tal fato explica a existência de portadores renais, fator primordial na epidemiologia da leptospirose, sendo que a transmissão ocorre pela exposição à urina de animais infectados e ambientes contaminados pela mesma (ACHA; SZYFRES, 2003; PLANK; DEAN, 2000). 
Embora os seres humanos sejam considerados hospedeiros acidentais na cadeia epidemiológica da leptospirose, e que não existam relatos de qualquer associação com um sorovar em particular, são descritas diferenças entre as fontes de infecção no meio urbano e no meio rural, além da atividade ocupacional e recreacional, e fatores climáticos, aos quais os humanos estão expostos (LAU et al. 2016; MORGAN et al. 2002).

A cadeia epidemiológica da leptospirose envolve três elos: a fonte de infecção, as vias de transmissão e o hospedeiro suscetível, sendo que as medidas de prevenção e controle da enfermidade deverão ser dirigidas a todos os elos dessa cadeia (BROD; FEHLBERG, 1992). De acordo com Blanco et al. (2016), o conhecimento dos sorovares prevalentes e seus hospedeiros de manutenção é essencial para o entendimento da cadeia epidemiológica da doença em qualquer área do mundo.

Segundo Jin et al. (2009), as bactérias são capazes de sobreviver no interior dos macrófagos e escapar da indução da apoptose, capacidade relacionada à virulência. A menos que ocorra a presença de anticorpos específicos, as leptospiras patogênicas são capazes de resistir à fagocitose por macrófagos e neutrófilos (ADLER, 2014). Assim, diferentes consequências da infecção aguda estariam relacionadas aos diferentes resultados da interação da Leptospira sp. com macrófagos (LI et al., 2007).

\section{DIAGNÓSTICO}

Como diagnóstico laboratorial a principal ferramenta é o teste de aglutinação microscópica (MAT), sendo a técnica padrão para o diagnóstico da leptospirose (ADLER, 2014). Na técnica, antígenos vivos reagem com amostras de soro e a aglutinação resultante é examinada através da microscopia de campo escuro (MUSSO; SCOLA, 2013). O teste depende da presença de quantidades detectáveis de anticorpos anti-Leptospira em amostras de pacientes convalescentes (PICARDEAU et al., 2014).

A Organização Mundial de Saúde (OMS) recomenda a utilização de um painel de cepas considerado padrão. A inclusão de um determinado sorovar no painel de antígenos vai depender do laboratório encarregado do teste (BLANCO et al., 2016), e o painel deverá conter amostras representando os sorovares de circulação local (PINTO et al., 2015), além dos mais prevalentes na espécie testada. Testes moleculares, como a reação em cadeia da 
polimerase (PCR) demonstram a presença do agente na amostra clínica e permitem a confirmação da doença durante os primeiros dias após a apresentação dos sintomas, uma das principais limitações do MAT (PICARDEAU et al., 2014).

A visualização direta de leptospiras em microscópio de campo escuro tem sido utilizada principalmente em amostras de urina durante a fase de leptospirúria, este exame, quando realizado imediatamente após a colheita da urina aumenta as chances de um resultado positivo, uma vez que o diagnóstico é baseado na morfologia e motilidade das espiroquetas (FAINE et al., 1999). Este teste apresenta como principais limitações: baixa sensibilidade, necessidade de observador experiente para diferenciar leptospiras de artefatos presentes nas amostras e a eliminação intermitente de leptospiras pela urina (OLIVEIRA, 2008). O isolamento de leptospiras permite o diagnóstico definitivo da leptospirose e a identificação do sorovar infectante, dado importante para orientar ações destinadas ao controle e profilaxia da doença (FAINE et al., 1999; VASCONCELLOS et al., 1997).

\section{ANIMAIS DOMÉSTICOS E A Leptospira EM POPULAÇÕES RURAIS}

De acordo com Schneider et al. (2015) as populações rurais do Rio Grande do Sul, têm cerca de oito vezes maior risco de contrair leptospirose do que as populações urbanas, e os casos rurais estão principalmente em duas zonas, caracterizadas pela maior produção de tabaco (região central) e maior produção de arroz (região sul).

Um estudo realizado por Barcellos et al. (2003) de casos notificados em Pelotas no Rio Grande do Sul, apresentou uma incidência de 16,3 casos por 100 mil habitantes, uma frequência superior à média nacional de 3,5 casos por 100 mil habitantes. Já Schneider et al. (2015), também sobre casos notificados no Rio Grande do Sul, indicam uma incidência de 1,2 a 2,4 casos para cada 10 mil habitantes no município de Pelotas.

Em seu estudo Schneider et al. (2015) revelaram indícios de uma nova associação entre exposição-desfecho da leptospirose, que é a plantação de tabaco no RS. O principal fator relacionado a essa associação seria o pH do solo, já que para o crescimento do tabaco o solo deverá apresentar um pH entre 5,0 e 6,5 (FAO, 2013), semelhante ao pH ótimo do solo para a manutenção de alguns sorovares de Leptospira, que é um pH de 6,2 para a sobrevivência ao longo de sete semanas no ambiente (ACHA; SZYFRES, 2003). De acordo com Khairani-bejo 
et al. (2004) os requisitos de sobrevivência no solo podem variar entre os sorovares, como o caso do sorovar Hardjo, comumente associado aos ruminantes, o qual cresce em um pH entre 6,5 e 6,8 .

A associação entre os trabalhadores da cultura do arroz e a leptospirose já foi descrita na literatura (CAMINITI et al., 2011; WHO, 2003). Destacam-se os riscos profissionais associados à infecção por leptospirose, tais como longo período de exposição à água, a falta de uso de equipamento de proteção individual (EPI) e presença de feridas na pele (CAMINITI et al., 2011). Assim, a conscientização de empregados e empregadores quanto à importância do uso de EPI, pode vir a amenizar a facilidade de contato com a bactéria no Rio Grande do Sul, propiciada por longas horas de trabalho em lavouras e condições ambientais ideais para multiplicação das bactérias.

Homem et al. (2001) na Amazônia Oriental Brasileira revelaram uma prevalência sorológica em humanos de $32,8 \%$, no qual sorovares como Hardjo e Grippotyphosa estavam entre os mais importantes, apresentando $6 \%$ e $4,5 \%$ de prevalência, respectivamente. Além disso, o sorovar Hardjo também estava entre os mais frequentes nos bovinos analisados, corroborando com os dados epidemiológicos que mostram os bovinos como importantes transmissores desse sorovar para humanos. Um estudo realizado por Brod et al. (1995) sobre a prevalência da leptospirose em bovinos, na região Sul do Rio Grande do Sul, mostrou 41,49\% de soros reagentes, com predominância do sorovar Hardjo (82,25\%). Também Herrmann et al. (2012), realizaram um estudo com bovinos na mesma região, encontrando uma prevalência de $38,75 \%$, apresentando títulos acima de 100 , sendo o sorovar Hardjo o mais prevalente, entre outros que reagiram estavam: Pyrogenes, Australis e Pomona. Estes dados mostram a importância dos bovinos na manutenção da leptospirose no meio rural gaúcho, e a necessidade de estudos que incluam a população exposta nessa cadeia produtiva.

Alguns autores como Subharat (2010) e Salgado et al. (2014) afirmaram que o gado e a agricultura representam um papel importante como fator de risco ocupacional para leptospirose humana, mas destacam também o papel de animais selvagens como reservatórios de Leptospira. Atividades externas, agricultura e contato com animais foram 
significantemente associados com a soropositividade para leptospirose (SETHI et al., 2003). Na mesma linha, Heat e Johnson (1994) observaram que grupos de alto risco para leptospirose são trabalhadores de fazendas e de matadouros, já que ficam expostos às Leptospiras, principalmente aos sorovares Pomona e Hardjo, e concluíram que geralmente a prevalência de leptospirose na população humana é um reflexo de prevalência na população animal com a qual as pessoas têm contato.

No meio rural de Pelotas, um estudo realizado por Jouglard e Brod (2000), em 213 propriedades rurais, analisou 489 amostras sorológicas de caninos, encontrando 13 (2,66\%) animais positivos no MAT com os títulos variando de 50 a 800, com reações para os sorovares Icterohaemorrhagiae, Australis, Copenhageni e Canicola. De acordo com Faine et al. (1999), cães, assim como ratos e camundongos, são as importantes fontes de infeç̧ão de Leptospira para o homem. Querino et al. (2003) demonstraram que o acesso à rua proporciona aos cães inúmeras possibilidades de se infectarem com Leptospira pelo contato direto ou indireto com outros animais, ou pelo acesso às áreas alagadiças.

Zacarias et al. (2008) reforçaram o papel dos ratos como reservatório do sorovar Copenhageni e também colocam a urina de gado infectado como um fator de risco para a leptospirose humana. De acordo com Silva et al. (2015) práticas sanitárias precárias são amplamente observadas em propriedades rurais do Rio Grande do Sul, tais como lixo e acúmulo de lixo orgânico, falta de saneamento, falha no acondicionamento de alimentos e a qualidade da água negligenciada, promovendo a proliferação de ratos e animais silvestres de vida livre, facilitando a transmissão do agente etiológico para um hospedeiro suscetível.

\section{CONTROLE}

O controle da leptospirose animal deve assentar-se na integração de medidas profiláticas instituída nos três níveis da cadeia de transmissão: fontes de infecção (vertebrados infectados), vias de transmissão (água, solo e fômites contaminados) e hospedeiros suscetíveis (vertebrados não infectados e não imunizados) (OLIVEIRA, 2008). Aplicação de medidas de saneamento que incluam: destino adequado do lixo, armazenagem correta dos alimentos de uso humano e animal em instalações construídas a prova dos roedores, evitar a armazenagem de entulhos ou objetos em desuso que possam fornecer abrigo para tais 
animais e, finalmente, a aplicação dos métodos ofensivos, representados pelo uso racional dos diversos tipos de rodenticidas (SIMÕES et al., 2016).

A proteção específica dos animais suscetíveis é obtida com o uso de vacinas inativadas que contenham os sorovares de leptospiras mais prevalentes nos hospedeiros da região (SALLES; LILENBAUM, 2006). Eliminar o excesso de água livre, com o emprego de técnicas de drenagem e canalização dos cursos de água (SIMÕES et al., 2016), assim como destinar adequadamente os esgotos e águas servidas contribuindo para a redução do nível de contaminação ambiental (OLIVEIRA, 2008).

\section{CONCLUSÃO}

As populações rurais do Rio Grande do Sul, principalmente da região central e sul do estado, estão mais expostas e com mais chances de adquirirem a leptospirose quando comparadas as populações das zonas urbanas. Atividades agrícolas e pecuárias que envolvem longos períodos em contato com a água aumentam o risco de contaminação. Bovinos, cães, roedores e outros animais silvestres infectados pela Leptospira são importantes para a manutenção da bactéria, representando elos importantes na cadeia de transmissão aos humanos. Falhas no saneamento e destino do lixo favorecem a presença de roedores nas propriedades, elevando o risco para a ocorrência da doença. Sugere-se que medidas como: reeducação da população rural, uso de EPI nas práticas agrícolas, manejo correto de resíduos e alimentos e vacinação dos animais domésticos suscetíveis poderiam diminuir os casos da doença em humanos, consideravelmente. 


\section{HUMAN LEPTOSPIROSIS: A REVIEW ABOUT THE DISEASE AND RISK FACTORS ASSOCIATED WITH RURAL POPULATIONS}

\section{ABSTRACT}

eptospirosis is a zoonosis of worldwide distribution and the transmission occurs through direct or indirect contact with carriers. The infection is caused by bacteria of the genus Leptospira and it is a disease of both humans and animals. Rural populations tend to be closer with animals, both wild and domestic, which increases the chance of contact with the agent. Thus, this study reviews literature regarding leptospirosis in the rural areas of the Rio Grande do Sul state (RS), Brazil, in the last 25 years and infers on the risks associated with farming activities. We demonstrate that rural populations, mainly in the southern and central regions of the state, have more risk of acquiring the disease, when compared to urban dwellers. In RS, activities associated with long work hours and lack of use of individual protection equipment, facilitate the contact with the bacteria. Among domestic animals, dogs and cattle are important for the maintenance and transmission of leptospirosis in these regions. Furthermore, sanitary shortcomings such as lack of proper sanitation and waste disposal favor the presence of rodents near the houses, another possible risk factor. This way, education regarding the disease, and proper use of individual protection equipment, seem to be the most needed measures to reduce human infection in the rural areas of the Rio Grande do Sul state, Brazil.

Keywords: Leptospira. Public health. Occupational disease. Zoonosis.

\section{LEPTOSPIROSIS HUMANA: UNA REVISIÓN SOBRE LA ENFERMEDAD Y LOS FACTORES DE RIESGO ASOCIADOS A LA ZONA RURAL}

\section{RESUMEN}

a leptospirosis es una zoonosis de distribución mundial cuya transmisión a las especies susceptibles ocurre por el contacto directo o indirecto con los enfermos. La enfermedad es causada por bacterias del género Leptospira, la cual ocurre tanto en humanos como en animales. En el medio rural, el riesgo de los humanos en adquirir la enfermedad es mayor que para los habitantes del medio urbano, principalmente por el contacto con animales domésticos, sinantrópicos y silvestres. En este contexto, este trabajo tuvo como objetivo revisar los principales estudios epidemiológicos sobre la leptospirosis en el medio rural del Estado del Rio Grande do Sul (RS) en los últimos veinticinco años, infiriendo sobre la influencia de las actividades agropecuarias en los casos de la enfermedad en humanos. Se evidencia que las poblaciones rurales en el RS, principalmente las localizadas en la región sur y central están más expuestas y tienen mayores posibilidades de contraer la leptospirosis, cuando son comparadas con la zona urbana. En RS, las actividades que involucran largos períodos de trabajo en contacto con el agua y la indisponibilidad, la mayoría de las veces, de equipos de protección individual (EPI), aumentan el riesgo para la leptospirosis. Entre los animales domésticos, se destacan los caninos y bovinos como 
importantes transmisores de la leptospirosis para los humanos en el medio rural. Además, fallas en el saneamiento y recolección de basura favorecen la presencia de roedores en las propiedades, elevando el riesgo para la ocurrencia de la enfermedad. Así, medidas como la reeducación, la información sobre los principales factores de riesgo de la enfermedad y el uso de EPI pueden ser utilizadas para prevenir casos graves de la leptospirosis en el medio rural del RS.

Palabras clave: Leptospira. Salud pública. Enfermedad ocupacional. Zoonosis.

\section{REFERÊNCIAS}

ACHA, P. N.; SZYFRES, B. Zoonoses and Communicable Diseases Common to Man and Animals: Bacterioses and Mycoses. 3. ed. Washington: Pan American Health Organization, vol. 1, p. 157-168, 2003.

ADLER, B. Pathogenesis of leptospirosis: cellular and molecular aspects. Veterinary Microbiology, v. 172, p .353-358, 2014.

BARCELLOS, C.; LAMMERHIRT, C. B.; ALMEIDA, M. A. B.; et al. Distribuição espacial da leptospirose no Rio Grande do Sul, Brasil: recuperando a ecologia dos estudos ecológicos. Cadernos de Saúde Pública, v. 19, n. 5, p. 1283-1292, 2003.

BHARTI, A. R.; NALLY, J. E.; RICALDI, J. N.; et al. Leptospirosis: a zoonotic disease of global importance. Lancet Infectious Diseases, v. 3, n. 12, p. 757-771, 2003.

BLANCO, R. M.; SANTOS, L. F.; GALLOWAY, R. L.; et al. Is the microagglutination test (MAT) good for predicting the infecting serogroup for leptospirosis in Brazil Comparative Immunology. Microbiology and Infectious Diseases, v. 44, p. 34-36, 2016.

BROD, C. S.; FEHLBERG, M. F. Epidemiologia da leptospirose em bovinos. Ciência Rural, v. 22, p. 239-245, 1992.

BROD, C. S.; MARTINS, L. F. S.; NUSSBAUN, J. R.; et al. Leptospirose bovina na região sul do Estado do Rio Grande do Sul. A Hora Veterinária, v. 14, p. 15-20, 1995.

CAMERON, C. E. Leptospiral structure, physiology and metabolism. Current Topics in Microbiology and Immunology, v. 387, p. 21-42, 2015.

CAMINITI, T. R.; ROMANI, R. F.; WONG, C. P.; et al. Prácticas laborales de riesgo en cultivadores de arroz del valle del Alto Mayo, Región San Martín, Perú. Revista Peruana de Epidemiologia, v. 15, p. 6, 2011.

COSTA, F.; HAGAN, J. E.; CALCAGNO, J.; et al. Global Morbidity and Mortality of Leptospirosis: A Systematic Review. PLOS Neglected Tropical Diseases, v. 9, n. 9, 2015. doi: 10.1371/journal.pntd.0003898. 
DREYFUS, A.; DYAL, J. W.; PEARSON, R.; et al. Leptospira Seroprevalence and Risk Factors in Health Centre Patients in Hoima District, Western Uganda. PLOS Neglected Tropical Diseases, v. 10, n. 8, p. 1-14, 2016. doi: 10.1371/journal.pntd.0004858.

FAINE, S. B.; ADLER, B.; BOLIN, C.; et al. Leptospira and Leptospirosis. Melbourne, Australia: MediSci, 1999. 272p.

FAO - Food and Agriculture Organization of the United Nations. Geo Network-geo-spatial data [Internet]. 2013. Disponível em:

<http://www.fao.org/geonetwork/srv/en/main.home>. Acesso em: 28 mar. 2017.

FIGUEIREDO, C. M.; MOURÃO, A. C.; OLIVEIRA, M. A.; et al. Leptospirose humana no município de Belo Horizonte, Minas Gerais, Brasil: uma abordagem geográfica. Revista da Sociedade Brasileira de Medicina Tropical, v. 34 n. 4, p. 331-338, 2001.

FONTES, A. P. A.; RIBEIRO, D. P.; JESUS, L. S. B.; et al. Aspectos funcionais respiratórios na leptospirose humana. Revista da Sociedade Brasileira de Medicina Tropical, v. 43, n. 2, p. 161-165, 2010.

GULATI, S.; GULATI, A. Pulmonary manifestations of leptospirosis. Lung India, v. 29, p. 347353, 2012.

HARTSKEERL, R. A.; COLLARES, P. M.; ELLIS, W. A. Emergence, control and re-emerging leptospirosis: dynamics of infection in the changing world. Clinical Microbiology and Infection, v. 17, p. 494-501, 2011.

HEAT, S. E.; JOHNSON, R. Leptospirosis. Journal of the American Veterinary Medical Association, v. 205, n. 11, p. 1518-1523, 1994.

HERRMANN, G. P.; RODRIGUES, R. O.; MACHADO, G.; et al. Soroprevalência de leptospirose em bovinos nas mesorregiões sudeste e sudoeste do estado Rio Grande do Sul, Brasil. Ciência Animal Brasileira, v. 13, n. 1, p. 131-138, 2012.

HOMEM, V. S. F.; HEINEMANN, M. B.; MORAES, Z. M. M.; et al. Estudo epidemiológico da leptospirose bovina e humana na Amazônia oriental brasileira. Revista da Sociedade Brasileira de Medicina Tropical, v. 34, n. 2, p. 173-180, 2001.

JIN, D.; OJCIUS, D. M.; SUN, D.; et al. Leptospira interrogans induces apoptosis in macrophages via caspase- 8 and caspase- 3 dependent pathways. Infection and Immunity, $v$. 77, p. 799-809, 2009.

JOUGLARD, S. D. D.; BROD, C. S. Leptospirose em cães: prevalência e fatores de risco no meio rural do Município de Pelotas, RS. Arquivos do Instituto de Biologia, v. 67, n. 2, p. 181$185,2000$. 
KHAIRANI-BEJO, S.; BAHAMAN, A. R.; ZAMRI-SAAD, M.; et al. The survival of Leptospira interrogans serovar Hardjo in the Malaysian environment. Journal of Animal and Veterinary Advances, v. 3, p. 123-129, 2004.

KO, A. I.; GOARANT, C.; PICARDEAU, M. Leptospira: the dawn of the molecular genetics era for an emerging zoonotic pathogen. Nature Reviews Microbiology, v. 7, p. 736-747, 2009.

LAU, C. L.; WATSON, C. H.; LOWRY, J. H.; et al. Human Leptospirosis Infection in Fiji: An Ecoepidemiological Approach to Identifying Risk Factors and Environmental Drivers for Transmission. PLOS Neglected Tropical Diseases, v. 10, n. 1, p. 1-25, 2016. doi: https://doi.org/10.1371/journal.pntd.0004405

LI, L.; OJCIUS, D. M.; YAN, J. Comparison of invasion of fibroblastos and macrophages by high and low-virulence Leptospira strains: colonization of the host-cell nucleus and induction of necrosis by the virulent strain. Archives Microbiology, v. 188, p. 591-598, 2007.

MORGAN, J.; BORNSTEIN, S. L.; KARPATI, A. M.; et al. Outbreak of Leptospirosis among Triathlon Participants and Community Residents in Springfield, Illinois, 1998. Clinical Infectious Diseases, v. 34, p. 1593-1599, 2002.

MUSSO, D.; SCOLA, B. Laboratory diagnosis of leptospirosis: a challenge. Journal of Microbiology, Immunology and Infection, v. 46, p. 245-252, 2013.

OLIVEIRA, F. C. S. Leptospirose bovina no Estado da Bahia, Brasil. Prevalência de sorovares predominantes, distribuição espacial e fatores de risco. São Paulo: USP, 2008. 123p. Dissertação (Mestrado em Veterinária), Faculdade de Medicina Veterinária e Zootecnia, Universidade de São Paulo, 2008.

PICARDEAU, M.; BERTHERAT, E.; JANCLOES, M.; et al. Rapid tests for diagnosis of leptospirosis: current tools and emerging technologies diagnostic. Microbiology and Infectious Disease, v. 78, n. 1, p. 1-8, 2014.

PINTO, P. S.; LOUREIRO, A. P.; PENNA, B.; et al. Usage of Leptospira spp. local strains as antigens increases the sensitivity of the serodiagnosis of bovine leptospirosis. Acta Tropica, v. 149, p. 163-167, 2015.

PLANK, R.; DEAN, D. Overview of the epidemiology, microbiology, and pathogenesis of Leptospira spp. In humans. Microbes and Infection, v. 2, p. 1265-1276, 2000.

QUERINO, A. M. V.; DELBEM, Á. C. B.; OLIVEIRA, R. C.; et al. Risk factors associated to leptospirosis in dogs in Londrina City - PR. Semina: Ciências Agrárias, v. 24, n. 1, p. 27-34, 2003. 
SALLES, R. S.; LILENBAUM, W. Leptospirose bovina no Brasil. Revista CFMV, v. 21, p. 42-46, 2006.

SALGADO, M.; OTTO, B.; SANDOVAL, E.; et al. A cross sectional observational study to estimate herd level risk factors for Leptospira spp. serovars in small holder dairy cattle farms in southern Chile. BMC Veterinary Research, v. 10, n. 126, p. 1-6, 2014.

SCHNEIDER, M. C.; NAJERA, P.; PEREIRA, M. M.; et al. Leptospirosis in Rio Grande do Sul, Brazil: Na Ecosystem Approach in the Animal-Human Interface. PLOS Neglected Tropical Diseases, v. 9, n. 11, 2015. doi.org/10.1371/journal.pntd.0004095

SETHI, S.; SOOD, A.; POOJA; et al. Leptospirosis in northern India: a clinical and serological study. Southeast Asian. Journal of Tropical Medicine and Public Health, v. 34, n. 4, p. 822825, 2003.

SILVA, F. J.; SILVA, G. C. P.; LOFFLER, S. G.; et al. Isolation of Leptospira spp. from a man living in a rural area of the Municipality of Cruz Alta, RS, Brazil. Ciência Rural, v. 45, n. 1, p. 47-51, 2015.

SIMÕES, L. S.; SASAHARA, T. H. C.; FAVARON, P. O.; et al. Leptospirose - Revisão. Pubvet, v. 10, n. 2, p. 138-146, 2016.

SUBHARAT, S. Epidemiology, diagnosis and vaccination control of leptospirosis in farmed deer in New Zealand. New Zealand: MUPN, 2010, 271p. Thesis (Doctor of Philosophy in Veterinary Clinical Science), Massey University Palmerston North, 2010.

VASCONCELLOS, S. A.; BARBARINI JÚNIOR, O.; UMEAHARA, O.; et al. Leptospirose bovina. Níveis de ocorrência e sorotipos predominantes em rebanhos dos Estados de Minas Gerais, São Paulo, Rio de Janeiro, Paraná, Rio Grande do Sul. Período de janeiro a abril de 1996. Arquivos do Instituto Biológico, v. 64, n. 2, p. 7-15, 1997.

ZACARIAS, F. G. S.; VASCONCELLOS, S. A.; ANZAI, E. K.; et al. Isolation of leptospira Serovars Canicola and Copenhageni from cattle urine in the state of Paraná, Brazil. Brazilian Journal of Microbiology, v. 39, n. 4, p. 484-488, 2008.

WHO - WORLD HEALTH ORGANIZATION. Human Leptospirosis: Guidance for Diagnosis, Surveillance and Control. Malta: WHO, 2003. 109p. 